\title{
BMJ Open Mental disorder in children with physical conditions: a pilot study
}

\author{
Alexandra Butler, ${ }^{1}$ Ryan J Van Lieshout, ${ }^{2}$ Ellen Louise Lipman, ${ }^{2}$ \\ Harriet L MacMillan, ${ }^{2}$ Andrea Gonzalez, ${ }^{2}$ Jan Willem Gorter, ${ }^{3}$ Kathy Georgiades, ${ }^{2}$ \\ Kathy N Speechley, ${ }^{4,5}$ Michael H Boyle, ${ }^{2}$ Mark A Ferro ${ }^{1}$
}

To cite: Butler A, Van Lieshout RJ, Lipman EL, et al. Mental disorder in children with physical conditions: a pilot study. BMJ Open 2018;8:e019011. doi:10.1136/ bmjopen-2017-019011

- Prepublication history for this paper is available online. To view these files, please visit the journal online (http://dx.doi. org/10.1136/bmjopen-2017019011).

Received 4 August 2017 Accepted 23 November 2017

\section{CrossMark}

${ }^{1}$ School of Public Health and Health Systems, University of Waterloo, Waterloo, Ontario,

Canada

${ }^{2}$ Department of Psychiatry and Behavioural Neurosciences, McMaster University, Hamilton, Ontario, Canada

${ }^{3}$ Department of Pediatrics, McMaster University, Hamilton, Ontario, Canada

${ }^{4}$ Department of Paediatrics, Western University, London, Ontario, Canada

${ }^{5}$ Department of Epidemiology and Biostatistics, Western University, London, Ontario, Canada

Correspondence to

Dr Mark A Ferro;

mark.ferro@uwaterloo.ca

\section{ABSTRACT}

Objectives Methodologically, to assess the feasibility of participant recruitment and retention, as well as missing data in studying mental disorder among children newly diagnosed with chronic physical conditions (ie, multimorbidity). Substantively, to examine the prevalence of multimorbidity, identify sociodemographic correlates and model the influence of multimorbidity on changes in child quality of life and parental psychosocial outcomes over a 6-month follow-up.

Design Prospective pilot study.

Setting Two children's tertiary-care hospitals.

Participants Children aged 6-16 years diagnosed in the past 6 months with one of the following: asthma, diabetes, epilepsy, food allergy or juvenile arthritis, and their parents. Outcome measures Response, participation and retention rates. Child mental disorder using the Mini International Neuropsychiatric Interview at baseline and 6 months. Child quality of life, parental symptoms of stress, anxiety and depression, and family functioning. All outcomes were parent reported.

Results Response, participation and retention rates were $90 \%, 83 \%$ and $88 \%$, respectively. Of the 50 children enrolled in the study, the prevalence of multimorbidity was $58 \%$ at baseline and $42 \%$ at 6 months. No sociodemographic characteristics were associated with multimorbidity. Multimorbidity at baseline was associated with declines over 6 months in the following quality of life domains: physical well-being, $\beta=-4.82(-8.47,-1.17)$; psychological well-being, $\beta=-4.10(-7.62,-0.58)$ and school environment, $\beta=-4.17(-8.18,-0.16)$. There was no association with parental psychosocial outcomes over time.

Conclusions Preliminary evidence suggests that mental disorder in children with a physical condition is very common and has a negative impact on quality of life over time. Based on the strong response rate and minimal attrition, our approach to study child multimorbidity appears feasible and suggests that multimorbidity is an important concern for families. Methodological and substantive findings from this pilot study have been used to implement a larger, more definitive study of child multimorbidity, which should lead to important clinical implications.

\section{INTRODUCTION}

The global prevalence of children with a chronic physical condition-a health

\section{Strengths and limitations of this study}

This pilot study includes children newly diagnosed with chronic physical conditions.

- This is the first study to examine mental disorder in children newly diagnosed with a number of different conditions.

- This study was likely underpowered to detect differences within and between children with and without multimorbidity and the small sample size limits generalisability.

- All outcomes were parent-reported.

problem that requires ongoing management over a period of years or decades-is common, affecting approximately $25 \%$ of children and increasing. ${ }^{1}$ These children may be adversely affected by their disorders or their treatment, subsequently developing additional conditions, including mental disorders. ${ }^{2}$ Compared with their peers with a physical or mental disorder only, children with multimorbidity (physical-mental comorbidity) experience greater symptom severity and impairment in both physical and mental health domains. ${ }^{3}$ Understandably, the onset of multimorbidity remains an important concern for children, parents, health professionals and payers. ${ }^{4}$

Mental disorders of any type are common in children and adolescents 5 and disproportionally affect young people with chronic physical conditions (herein physical conditions). ${ }^{6} 7$ Estimates from clinical samples suggest that nearly half of the children with physical conditions meet criteria for a mental disorder diagnosis. ${ }^{8}$ In general population samples, this estimate is lower, with approximately $20 \%-30 \%$ of children being affected. ${ }^{3}$

Evidence of the association between physical and mental health is robust, ${ }^{9-11}$ but with few exceptions, ${ }^{7} 1213$ the literature base consists mostly of cross-sectional studies. This limits our understanding of mental health at the time children are 
diagnosed with a physical condition and how their mental health may change over time. One study found more parent-reported symptoms of problem behaviour in children aged 6-7 years newly diagnosed with a chronic illness compared with healthy controls. ${ }^{7}$ This effect was stable through to 10-11 years of age, highlighting the chronicity of multimorbidity in childhood. In a prospective study of children with diabetes, HRs for mental disorder were highest within 6 months of the diabetes diagnosis (3.0 (2.7-3.4)) compared with those with a duration of diabetes of 5 years or more (1.9 (1.7-2.1)), regardless of age at diagnosis, ${ }^{12}$ suggesting that the peridiagnostic period may be a time of particular mental health risk. Another prospective study showed changes over time in associations of mental health with physical conditions being associated with depressive symptoms during childhood, and with anxiety symptoms during early adolescence. ${ }^{13}$ While these studies have advanced the field, opportunities exist to overcome the limitations in these studies associated with the ascertainment of physical conditions based on parent report, ${ }^{14}$ assessment of symptoms of problem behaviours rather than mental disorder ${ }^{131516}$ and inherent weaknesses of using register-based data related to data quality and variable availability. ${ }^{17}$

Existing research also suggests that physical conditions and mental disorders are independently associated with poorer psychosocial outcomes including quality of life $\mathrm{e}^{1819}$ and self-esteem, ${ }^{2021}$ as well as academic performance. ${ }^{2223}$ These adverse effects can also extend to parents and families who experience elevated stress and psychological distress, worse family functioning and financial hardship. ${ }^{24-28}$ Effects on child and parent psychosocial outcomes appear similar when physical and mental disorders are examined separately; however, there is little research examining whether multimorbidity exerts a compounding effect. Cross-sectional evidence suggests that children with multimorbidity experience worse quality of life compared with children with a physical or mental disorder alone. ${ }^{29}{ }^{30}$ One prospective study showed that adults who experienced multimorbidity during adolescence had lower quality of life compared with those who had a physical or mental disorder only. ${ }^{31}$ These researchers found that among those with multimorbidity, physical conditions affected physical quality of life only, while their mental disorder negatively affected multiple domains of life quality, including physical, emotional and social well-being. The extent to which multimorbidity influences other aspects of parental health and wellbeing, including parenting stress, psychopathology and family relationships, is not well known.

Despite the progress made in understanding child multimorbidity and its effects on psychosocial outcomes, important knowledge gaps remain. First, the burden and correlates of multimorbidity, particularly in clinical samples of children who represent the largest consumers of health services ${ }^{32}$ is not well known. While other studies have examined prevalence of multimorbidity, those studies were based on population, not clinical samples of prevalent cases and did not measure Diagnostic and Statistical Manual of Mental Disorders (DSM)-aligned diagnoses ${ }^{3}{ }^{13}$, were out-dated ${ }^{8}$ or focused on a single physical condition. ${ }^{12}$ This information is needed to inform resource allocation and the provision of services within the health system. Second, the timing of multimorbidity onset, how it changes and its influence on psychosocial outcomes over time are not well understood, limiting our ability to identify opportunities for intervention to prevent the development of mental disorder in children with physical conditions. This includes a lack of understanding how mental disorders may change or appear in relation to the onset of the physical condition. For example, are anxiety disorders more common at the time of diagnosis given the uncertainty surrounding prognosis? Third, effects of child multimorbidity on parental health and well-being have not been explored in much detail. Understanding these effects is key to designing, implementing and evaluating family-centred approaches to care within the paediatric setting to promote the best possible health outcomes for children, parents and families. ${ }^{33}$

Anticipating substantial hardship, stress and psychological distress associated with receiving a diagnosis of a physical condition in childhood within families, as well as prognostic uncertainty, ${ }^{12}{ }^{34-38}$ we conducted a pilot study to assess the feasibility of recruitment of eligible participants, estimating respondent burden related to data collection, and the extent of missing data and attrition. Substantively, the aims of the pilot study were to (1) examine the initial prevalence of multimorbidity in a clinical sample of children newly diagnosed with a physical condition, as well as rates 6 months later; (2) identify correlates of multimorbidity in children and parents and (3) explore the influence of multimorbidity on changes in child quality of life and parental psychosocial outcomes over 6 months of follow-up. Based on previous clinical studies, ${ }^{8}$ we hypothesised that at the time of diagnosis, $50 \%$ of children would screen positive for mental disorder. Based on limited evidence, ${ }^{12}$ we hypothesised that 6 months later, there would be a decrease in the proportion of multimorbidity. Finally, we hypothesised that children with multimorbidity would have worse quality of life over time; their parents, more symptoms of parenting stress, anxiety and depression; and their families, worse functioning compared with children with physical conditions only.

\section{METHODS}

\section{Sample}

Data come from a multisite, prospective, pilot study aimed at examining mental disorder(s) in children with physical conditions. Families were recruited from four outpatient clinics from two paediatric academic hospitals (specialised tertiary care centres; two clinics per hospital) in Ontario, Canada, to assess mental and psychosocial outcomes in children with newly diagnosed 
physical conditions. Health professionals at the hospitals were involved at the initial point of contact and provided eligible families with an overview of the study and details regarding participation. The eligibility criteria for the study were children who (1) were aged $6-16$ years $(6$ is the youngest age at which our measure of mental disorder is validated; the ceiling age of 16 years ensured that during the follow-up, participants did not transfer out of the paediatric health system); (2) had received a diagnosis of asthma, diabetes, epilepsy, food allergy or juvenile idiopathic arthritis (which represent the most common physical conditions among children) ${ }^{39}$ within the 6 months prior to recruitment and (3) had a parent who could read English (not all measures have been validated in other languages). Children were excluded if they had a degenerative neurological disorder because child and parental outcomes are well established in this population. Child IQ was not tested and children were not excluded if their parents indicated intellectual disability, maximising the coverage and representativeness of our sample. Following sample size guidelines suggested for the conduct of pilot studies ${ }^{40}$ we aimed to recruit 60 children and families (12 per condition) over a 12-month period.

\section{Data collection}

After the medical encounter, eligible families were invited by clinic nurses to speak with research staff about the study. Research staff briefly introduced the study and provided families an information letter. Families interested in participating in the study consented for clinic nurses to send their contact information to study investigators who then followed up with families by telephone to confirm eligibility, obtain oral consent from parents and children and arrange for a convenient time to conduct a telephone interview to assess child mental health. Parents also completed two mailed surveys to assess psychosocial outcomes and demographic characteristics: one at baseline and one 6 months later, when a second telephone interview to assess mental health was conducted. Parents of all participating children provided proxy reports and children who were $\geq 11$ years of age $(n=33)$ self-reported on the telephone interview and to the mail survey. Parents and children also consented to have health professionals provide clinical information at the same measurement occasions.

\section{Measures}

Mental disorder

Child mental disorder(s) were assessed using the Mini International Neuropsychiatric Interview for Children and Adolescents (MINI-KID). ${ }^{41}$ The MINI-KID is a structured diagnostic interview used to assess DSM-IV disorders in children aged 6-17 years and has been validated against the Schedule for Affective Disorders and Schizophrenia for School Aged Children-Present and Lifetime Version. ${ }^{41}$ It is composed of diagnostic modules that contain screening questions and skip patterns for each disorder. Phone interviews were administered separately: the MINI-KID(c), to children $\geq 11$ years and the MINI-KID (p) (proxy version), to all parents at both measurement occasions. The MINI-KID was administered by a single interviewer who underwent training that included monitored practice. The presence of the most common mental disorders was assessed: major depressive episode, separation anxiety disorder, social phobia, specific phobia, generalised anxiety disorder, attention deficit hyperactivity disorder, oppositional defiant disorder and conduct disorder. ${ }^{42}$ The MINI-KID has demonstrated strong test-retest reliability compared with other instruments. ${ }^{15}$ Mental disorder was classified according to parent reports on the MINI-KID.

\section{Quality of life}

Child quality of life between the two visits was measured using the KIDSCREEN-27, ${ }^{43}$ a 27-item child and parent-reported generic measure that assesses five domains: physical well-being (five items; examines physical activity and energy), psychological well-being (seven items; examines emotional balance and life satisfaction), autonomy and parent relations (seven items; examines family dynamics and age-appropriate freedoms), social support and peers (four items; examines nature of peer relationships) and school environment (four items; examines perception of cognition, learning and feelings about school). Responses are scored using a fivepoint Likert scale, and domain scores are transformed into T-scores with a mean of 50 and an SD of 10 (higher scores indicate better quality of life). The KIDSCREEN-27 has been found to be valid and reliable in children with and without physical conditions, ${ }^{43} 44$ and demonstrated adequate agreement between children and parents. ${ }^{45}$ Internal consistency reliabilities for each domain from this study were good for both child $(\alpha=0.75-0.89)$ and parent reports $(\alpha=0.83-0.92)$.

\section{Parental stress}

The Parental Stress Scale (PSS) measures parental stress across the domains of rewards, stressors, loss of control and satisfaction. ${ }^{46}$ The 18 items are rated on a five-point Likert scale (eight items are reverse-coded) with higher scores (range: 18-90) indicating more parental stress. The psychometric properties of the PSS are robust: testretest reliability $(\mathrm{r}=0.81)$ and convergent validity with the Parenting Stress Index $(r=0.75)$ and Perceived Stress Scale $(r=0.41) .{ }^{46}$ Internal consistency for the PSS in this study was $\alpha=0.84$.

\section{Parental anxiety}

The State Trait Anxiety Inventory (STAI) is a widely used tool for measuring anxiety. Of the 40 questions in the STAI survey, we considered 'trait anxiety' items only that aim to measure how parents generally feel, as well as their propensity for perceived anxiety. ${ }^{47}$ Survey responses were scored from 1 to 4 (seven items are reverse-coded). Scores were summed together (range: 20-80) with higher scores indicating higher levels of anxiety. The STAI has 
robust psychometric properties, with trait-specific testretest reliabilities of $\mathrm{r}=0.73-0.86$, and has been shown to be valid with other questionnaires used to assess anxiety $(\mathrm{r}=0.73-0.85) .{ }^{47} 48$ In this study, internal consistency for the STAI was $\alpha=0.89$.

\section{Parental depression}

Parental symptoms of depression were measured with the Center for Epidemiological Studies Depression Scale (CES-D), a 20-item scale designed to assess depressive symptomatology in the general adult population over the past week. ${ }^{49}$ The CES-D includes items that survey the domains of positive and negative effect, somatic activity and interpersonal relations. A four-point Likert scale is used to rate the frequency of symptoms experienced. Higher scores (range: 0-60) indicate greater frequency of depressive symptoms, and individuals with total scores $\geq 16$ are typically identified as having clinically significant levels of depression. ${ }^{49}$ Extensive research has shown the CES-D to be valid and reliable. ${ }^{480}$ In this study, internal consistency for the CES-D was $\alpha=0.93$.

\section{Family functioning}

The 12-item General Functioning subscale of the McMaster Family Assessment Device (FAD) provided a valid and reliable measure of the health/pathology of the family (ie, family functioning)..$^{5152}$ The scale is derived by summing items from six domains: problem solving, communication, roles, affective responsiveness, affective involvement and behavioural control. Items are rated on a four-point Likert scale with higher scores (range: 0-36) indicating poorer overall family functioning. Internal consistency for the FAD in this study was $\alpha=0.92$.

\section{Physical condition disease severity}

Disease severity in children was assessed and measured by a health professional using a $10 \mathrm{~cm}$ Visual Analogue Scale (VAS). The VAS represents a continuum of disease severity. ${ }^{53}$ Health professionals marked the VAS at the point which best reflected the disease severity of the child, according to their clinical judgement. The distance from the zero point of the VAS (left side) to the mark was measured and recorded as the disease severity of the child. The VAS and its scoring method have been used in a variety of populations and settings to assess well-being and pain, and has the advantage of being easily comparable across study samples. ${ }^{545}$

\section{Demographic characteristics}

Sociodemographic data were collected on child and parent age, sex and immigrant status, parent marital status and educational attainment, as well as annual household income.

\section{Analysis}

Comparisons between children with and without multimorbidity were made using Mann-Whitney (continuous variables) and Fisher's exact tests (categorical variables). Changes in the prevalence of multimorbidity from baseline to 6 months was using the McNemar test. Analysis of variance with post hoc Scheffé tests was conducted to compare KIDSCREEN-27 scores with available population norms. ${ }^{43}$ Generalised linear modelling was used to examine the association between multimorbidity and children's quality of life and parent psychosocial outcomes at 6 months. Outcomes were regressed on presence of multimorbidity, controlling for baseline scores for each respective outcome, as well as child age, sex and physical condition. These covariates were included in the models to present unbiased estimates of effect. All analyses were conducted using SPSS V.21 (IBM). Due to the pilot nature of this study, statistical tests were two-tailed using a significance level of $\alpha=0.10$. As there were no missing item-level data, complete case analysis was used.

\section{RESULTS}

\section{Sample characteristics}

Over 12 months, 62 families were approached to participate in the study. Of these, 56 (90\% response) agreed to participate. Four families were not interested in participating and two families had a child that did not meet the eligibility criteria. Fifty families (83\% participation) completed the baseline assessment (telephone and mail) and forty-four (88\% retention) completed the 6-month follow-up (telephone and mail). Repeated attempts to contact the six families who completed the telephone interview, but did not return the complete mail survey, were unsuccessful; thus, the reasons for withdrawal from the study are unknown. There were no baseline differences between families lost to follow-up and those who completed the study.

Baseline characteristics are shown in table 1 . The mean age of children was 11.3 (SD 3.3) years and 52\% were male. There was no difference in the number of children across physical condition subgroup, though asthma was the most common (28\%) and epilepsy and food allergy, the least common (16\%). Parents had a mean of age of 44.0 (5.7) years and $90 \%$ were female. Most parents were Caucasian $(94 \%)$, married $(78 \%)$, had completed postsecondary education $(78 \%)$ and had annual household incomes of $\geq \$ C 90000(58 \%)$.

\section{Prevalence of multimorbidity}

The prevalence of parent-reported multimorbidity declined from $58 \%$ at baseline to $42 \%$ at 6 months $(\mathrm{P}=0.09$; table 2). At baseline, 11 (22\%) children had multiple mental disorders. This decreased to nine (18\%) at 6 months. Anxiety disorder (at least one of separation anxiety, phobias or generalised anxiety) was the most common disorder at baseline (36\%) and 6 months (26\%). Differences across physical conditions were found for the prevalence of attention-deficit hyperactivity disorder $\left(\chi^{2}=6.44 ; \quad \mathrm{P}=0.06\right)$ and oppositional-defiant disorder $\left(\chi^{2}=7.53 ; \mathrm{P}=0.07\right)$ at baseline and for attention-deficit hyperactivity disorder $\left(\chi^{2}=7.98 ; \mathrm{P}=0.09\right)$ at 6 months. In 
Table 1 Baseline sample characteristics

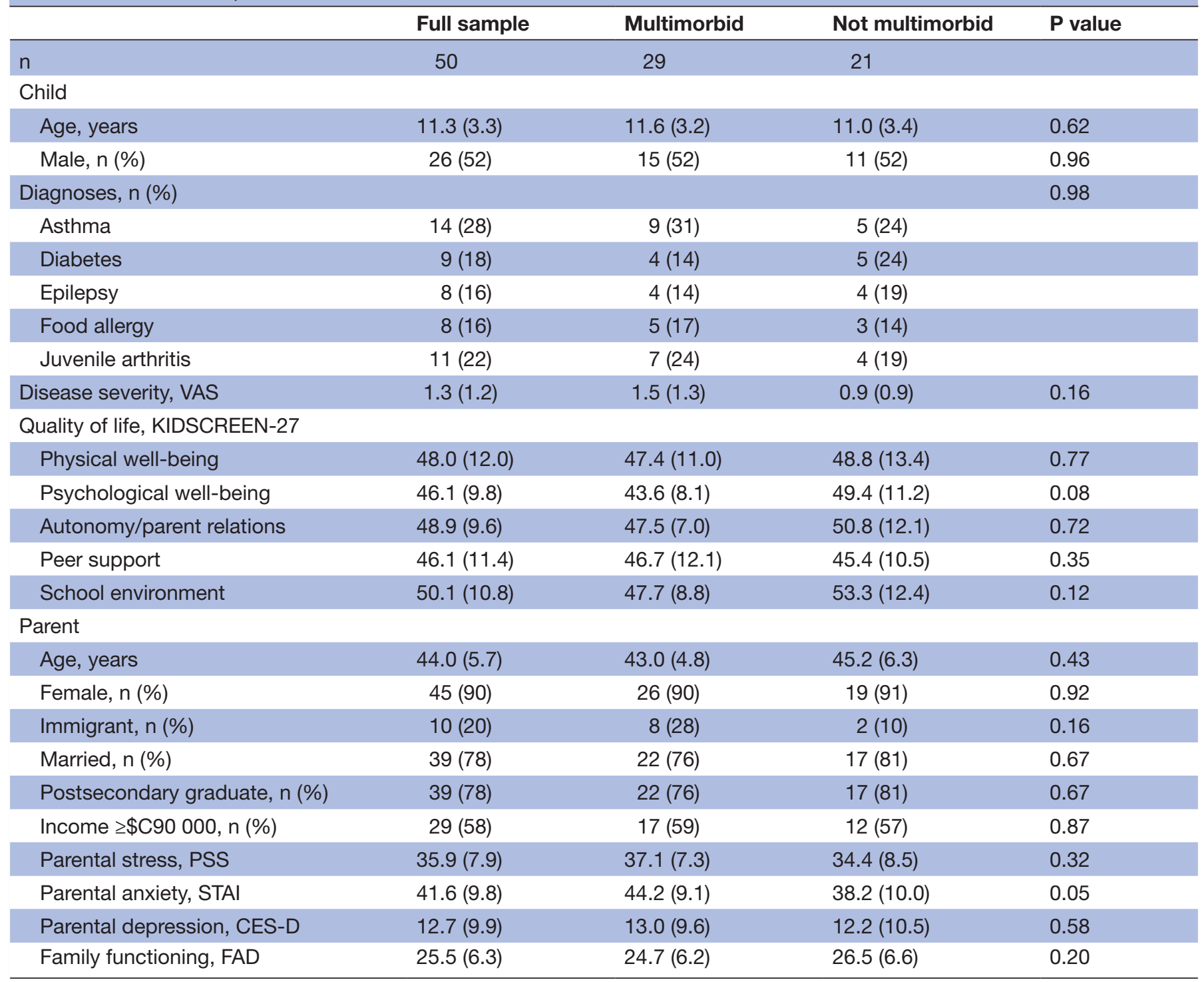

Results are reported as mean (SD) unless otherwise noted.

CES-D, Center for Epidemiological Studies Depression Scale; FAD, McMaster Family Assessment Device; PSS, Parental Stress Scale; STAI, State Trait Anxiety Inventory; VAS, Visual Analogue Scale.

each case, the proportion of mental disorder was elevated in children with food allergy.

The prevalence of child-reported multimorbidity was substantially lower than that reported by parents-18\% at baseline and $15 \%$ at 6 months. Given the low number of age eligible children to provide self-reports, differences in mental disorder across physical conditions were not examined. Parent-child agreement on the MINI-KID for any mental disorder was $\kappa=0.15$.

\section{Correlates of multimorbidity}

Results showed no differences in child and parent characteristics between children with and without parent-reported multimorbidity with two exceptions (table 1): children with multimorbidity had lower KIDSCREEN-27 psychological well-being (43.6 vs 49.4; $\mathrm{P}=0.08)$ and parents reported higher STAI scores (44.2 vs
38.2; $\mathrm{P}=0.05)$. Among children who provided self-reports, those with multimorbidity reported lower KIDSCREEN-27 scores in the following domains: psychological well-being (38.1 vs 49.1; $\mathrm{P}<0.01$ ), peer support (41.6 vs 50.7; $\mathrm{P}<0.01$ ) and school environment (41.8 vs 51.5; $\mathrm{P}=0.01$ ).

\section{Multimorbidity and psychosocial outcomes}

Comparisons of parent-reported KIDSCREEN-27 scores between our sample and population norms are shown in figure 1. Overall differences were found for the physical well-being, psychological well-being and peer support domains. Post hoc tests showed that compared with population norms, children with multimorbidity had significantly poorer psychological well-being $(\mathrm{t}=4.21 ; \mathrm{P}<0.01)$ and children without multimorbidity had lower peer support $(\mathrm{t}=2.66 ; \mathrm{P}<0.01)$. Results of the unadjusted and adjusted generalised linear models of the association of 
Table 2 Prevalence of multimorbidity

\begin{tabular}{|c|c|c|c|c|c|c|c|}
\hline & Full sample & Asthma & Diabetes & Epilepsy & Food allergy & $\begin{array}{l}\text { Juvenile } \\
\text { arthritis }\end{array}$ & $P$ value \\
\hline \multicolumn{8}{|l|}{ Baseline } \\
\hline $\begin{array}{l}\text { Major depressive } \\
\text { episode }\end{array}$ & $12(24)$ & $4(29)$ & $2(22)$ & 1 (13) & $1(13)$ & $4(36)$ & 0.74 \\
\hline Separation anxiety & $2(4)$ & 0 & 0 & $1(13)$ & 0 & $1(9)$ & 0.43 \\
\hline Generalised anxiety & $6(12)$ & 0 & 0 & $2(25)$ & $2(25)$ & $2(18)$ & 0.11 \\
\hline $\begin{array}{l}\text { Attention-deficit } \\
\text { hyperactivity }\end{array}$ & $5(10)$ & $1(7)$ & 0 & 1 (13) & $3(38)$ & 0 & 0.06 \\
\hline $\begin{array}{l}\text { Oppositional } \\
\text { defiant }\end{array}$ & $9(18)$ & $2(14)$ & $1(11)$ & $2(25)$ & $4(50)$ & 0 & 0.07 \\
\hline Any disorder & $21(42)$ & $9(64)$ & $1(11)$ & $4(50)$ & $2(25)$ & $5(56)$ & 0.10 \\
\hline $\begin{array}{l}\text { Major depressive } \\
\text { episode }\end{array}$ & $2(4)$ & 0 & 0 & 0 & 0 & $2(22)$ & 0.11 \\
\hline Separation anxiety & $3(6)$ & $2(14)$ & 0 & $1(13)$ & 0 & 0 & 0.56 \\
\hline Phobia* & $10(20)$ & $5(36)$ & $1(11)$ & $1(13)$ & 0 & $3(33)$ & 0.25 \\
\hline Generalised anxiety & $4(8)$ & $1(7)$ & 0 & $1(13)$ & 0 & $2(22)$ & 0.55 \\
\hline $\begin{array}{l}\text { Attention-deficit } \\
\text { hyperactivity }\end{array}$ & $3(6)$ & 0 & 0 & 0 & $2(25)$ & $1(11)$ & 0.09 \\
\hline $\begin{array}{l}\text { Oppositional } \\
\text { defiant }\end{array}$ & $6(12)$ & $2(14)$ & 0 & $2(25)$ & $2(25)$ & 0 & 0.31 \\
\hline
\end{tabular}

*Includes generalised, non-generalised and specific phobias.

Results are the number (\%) of children with multimorbidity. Fisher's exact tests examined multimorbidity across the five physical conditions.

parent-reported multimorbidity with quality of life over time are shown in table 3. Adjusting for child age, sex, type of physical condition and baseline KIDSCREEN-27 score, multimorbidity was associated with lower scores in the following domains at 6 months: physical well-being $(\mathrm{B}=-4.82 ; \mathrm{P}=0.03)$, psychological well-being $(\mathrm{B}=-4.10$; $\mathrm{P}=0.06)$ and school environment $(\mathrm{B}=-4.17 ; \mathrm{P}=0.09)$. With

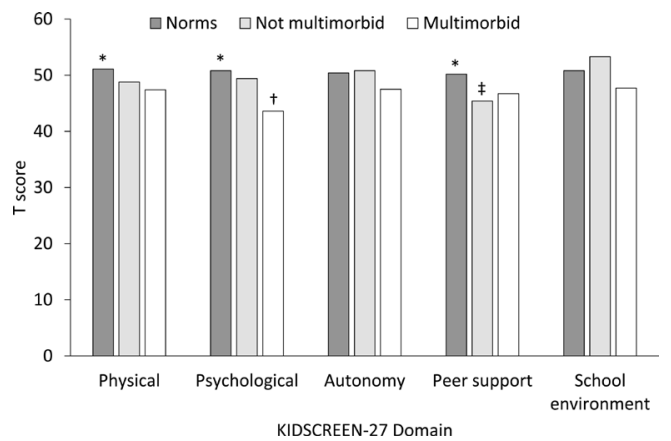

Figure 1 Comparison of KIDSCREEN-27 scores with population norms. ${ }^{*} \mathrm{P}<0.10$ for overall F-test across the three groups. †Multimorbid group significantly lower than normative and not multimorbid groups. $¥$ Not multimorbid group significantly lower than normative group. the exception of autonomy and parent relations, the strength of the association increased after covariate adjustment. Though similar estimates of association were found for child reports (multimorbidity and KIDSCREEN-27), only the association between multimorbidity and psychological well-being was statistically significant $(B=-10.66$; $\mathrm{P}=0.03)$.

The same modelling strategy was used to examine the associations with parental stress, anxiety, depression and family functioning. In both unadjusted and adjusted models, multimorbidity was not associated with any psychosocial outcomes in parents over time (table 4). Similarly, the strength of association (though not statistically significant) increased after covariate adjustment.

\section{DISCUSSION}

In this pilot study, over half of the children screened positive, based on parent report, for mental disorder(s) soon after being diagnosed with a physical condition, and this proportion appeared to decrease 6 months later. This contrasted self-reported mental disorder, in which approximately one in five children screened positive 
Table 3 Longitudinal effects of multimorbidity on child quality of life

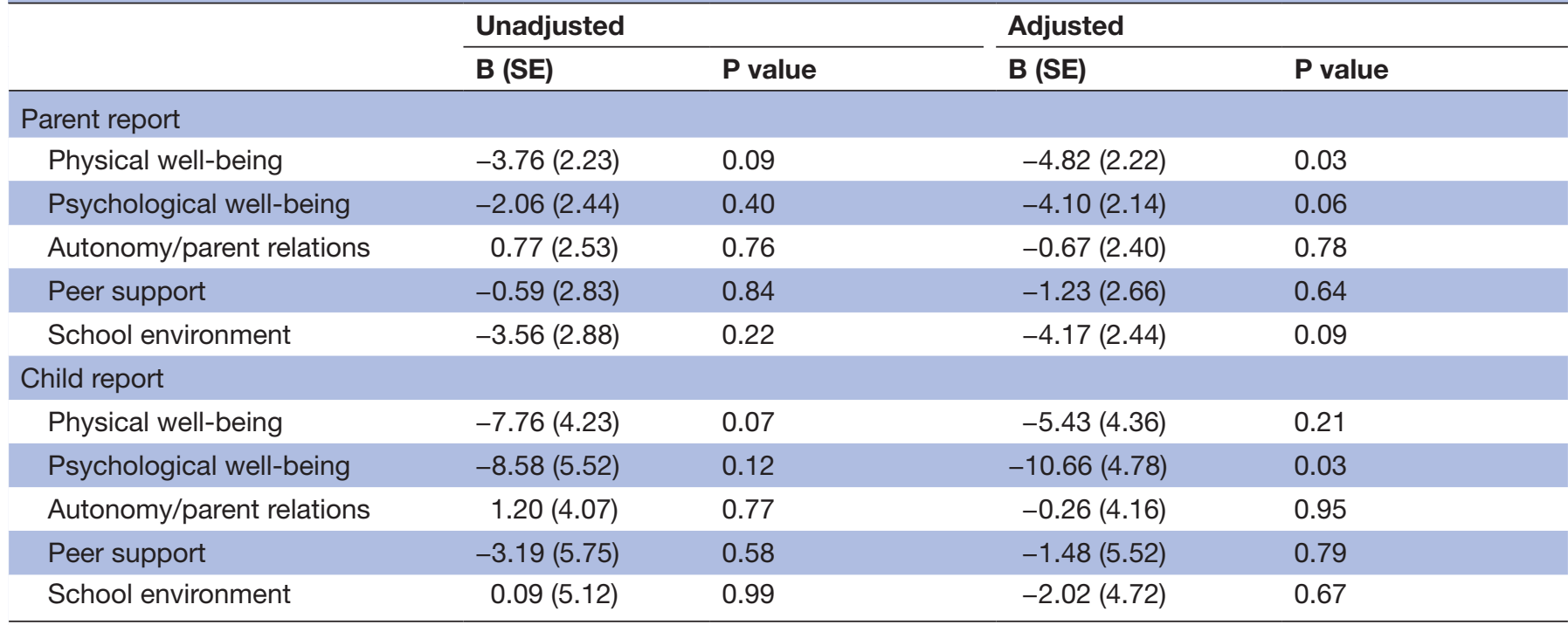

Parent models include parent-reported Mini International Neuropsychiatric Interview for Children and Adolescents (MINI-KID) and KIDSCREEN-27, whereas child models included child-reported MINI-KID and KIDSCREEN-27. Models adjusted for child age and sex, physical condition and baseline quality of life.

and which remained relatively stable over time. Anxiety disorders were found to be the most common disorders affecting children at diagnosis and 6 months later. There were no sociodemographic differences between children with and without multimorbidity. While multimorbidity did have a negative effect on child quality of life over time, our hypothesis that it would also influence parental outcomes was unsupported.

Methodologically, this pilot work has implications for the study of child multimorbidity within the clinical setting. Regarding participant recruitment, we limited the amount of contact between research staff and families during the initial contact in the clinic. This served two purposes: one, it minimised burden on the physicians and nurses whose primary focus is clinical care, as well as clinical staff managing large patient volumes. Two, it reduced the amount of information passed to families at a time when they may have felt overwhelmed with the clinical information provided by the physician about their child's diagnosis. We provided an information letter and then followed up by telephone a few days later when families were away from the clinic and had a chance to review this letter and determine if they wanted to participate. Our approach of engaging families personally in clinic, followed by telephone contact and data collection via mail survey, was found to be acceptable to families. Our strong response and retention rates contrast evidence showing reduced response rates in research studies. ${ }^{56}$ The majority of families in our study also noted that mail survey was the preferred method for data collection compared with online surveys and home interviews (data not shown). Overall, our methodology resulted in good coverage, with over $80 \%$ of consecutively approached eligible families participating in the study. This suggests that the mental health of children with physical conditions is an important concern for families, and that they are willing to contribute their time to such research studies. Our recruitment experience suggested that a number of children were ineligible for the study because their illness duration was greater than 6 months. To ensure a more efficient recruitment that encompasses an even larger coverage of our target population, our large-scale study will include children as young as 2 years of age, and we are expanding the number of physical conditions (eg, bowel diseases, chronic headache, lupus).

Table 4 Longitudinal effects of multimorbidity on parental outcomes

\begin{tabular}{lllll}
\hline & \multicolumn{3}{l}{ Unadjusted } & \multicolumn{1}{l}{ Adjusted } \\
\cline { 2 - 3 } \cline { 5 - 5 } & B (SE) & P value & B (SE) & P value \\
\hline Parental stress, PSS & $0.28(1.60)$ & 0.86 & $-1.50(1.51)$ & 0.32 \\
Parental anxiety, STAI & $0.14(2.05)$ & 0.95 & $-0.24(2.15)$ & 0.91 \\
Parental depression CES-D & $0.30(1.12)$ & 0.80 & $0.62(1.13)$ & 0.58 \\
Family functioning, FAD & $1.21(1.32)$ & 0.36 & $1.47(1.28)$ & 0.25 \\
\hline
\end{tabular}

Models adjusted for child age and sex, physical condition and baseline psychosocial outcome.

CES-D, Center for Epidemiological Studies Depression Scale; FAD, McMaster Family Assessment Device; PSS, Parental Stress Scale; STAI, State Trait Anxiety Inventory. 
Our study reaffirms the need to consider the perspective of multiple informants when assessing the presence of child mental disorder. ${ }^{5758}$ Parent-child agreement was nearly identical to previous research, ${ }^{58}$ suggesting that the presence of a physical condition in children does not appear to influence the level of agreement between child and parent reports of mental disorder. The extent to which the excess proportion of multimorbidity identified by parents is clinically relevant requires additional study that includes assessments by mental health professionals to verify clinical diagnoses of mental disorder.

Our estimate of the proportion of children with multimorbidity was similar to previous reports ${ }^{8}$ and supports the chronicity of multimorbidity during the early stages of being diagnosed with a physical condition. ${ }^{7}$ As shown in previous work in children with diabetes, ${ }^{12}$ the peridiagnostic period represents a critical developmental period for mental health. While this study did not measure mental disorder prior to the diagnosis of a physical condition, elevated rates of anxiety disorder at the time of diagnosis may be attributable to the uncertainty that children may experience (either before or after diagnosis) regarding the prognosis of their physical condition, including unpredictability of exacerbations, fear of death, loss of control, stigma associated with their condition or adverse effects of medical treatment. ${ }^{10}$ From this perspective, anxiety arises from negatively biased thought patterns that exaggerate adverse effects of the physical condition and can undermine confidence in adapting to threatening situations. ${ }^{59}$ Anxiety in these children may be an inherited trait or learnt behaviour-parents of children with multimorbidity in our sample reported more symptoms of anxiety compared with parents of children without multimorbidity. There is also emerging evidence of shared biological pathways that underlie multimorbidity. In adults, symptoms of anxiety are associated with systemic inflammation, ${ }^{60}$ which is elevated in individuals with physical conditions. Whether markers of inflammation, such as proinflammatory cytokines, mediate the relationship between physical and mental disorder is unknown.

These findings also contribute to the converging evidence that risk for mental disorder is relatively consistent among children with various physical conditions. ${ }^{61}$ One exception was that attention-deficit hyperactivity disorder was more common among children with food allergy. This increased risk is supported by some previous studies. ${ }^{37}$ As in this work, attention-deficit hyperactivity disorder in our sample of children with food allergy was mainly of the inattentive subtype. Inattentiveness may co-occur with core symptoms of generalised anxiety disorder, manifesting because of hypervigilance in avoiding food allergens. From a biological perspective, there is evidence of shared immunological ${ }^{63}$ and inflammatory ${ }^{64}$ responses for allergic conditions and attention-deficit hyperactivity disorder which may explain this association. Given the small number of children with food allergy in our sample, these interpretations are by no means definitive, but instead are offered as hypotheses to be tested rigorously in larger samples.

In general, the sample consisted of high socioeconomic two-parent families, which may have contributed to the lack of sociodemographic differences between children with and without multimorbidity and limits the generalisability of the findings. Placing the finding in the context of previous work is difficult, given the absence of studies examining sociodemographic correlates of multimorbidity. Previous population-based studies conducted in Canada also showed no socioeconomic differences between children with and without physical conditions. ${ }^{245-67}$ In our large-scale study, we will work towards a recruitment strategy that will include wider variation in the socioeconomic status to families to increase the representativeness of the sample. Contrary to expectation, no effect of multimorbidity on parental outcomes was found. Nevertheless, information related to parental psychopathology and family environment may be important control variables used to isolate the effects of multimorbidity on child outcomes. Such family processes may also be implicated in complex pathways linking physical and mental health in children. As a result, these variables will be included in the large-scale study.

Multimorbidity appears to have a negative effect on children's quality of life, above and beyond the effect of having a physical condition alone. ${ }^{18}$ This effect is pervasive, affecting multiple domains of quality of life during the first 6 months after a diagnosis. Of interest is the finding that the magnitude of effect seen for physical well-being, psychological well-being and school environment was approximately half an SD. This metric has been validated as the minimal clinically important difference for measures of quality of life ${ }^{68}$ and provides evidence to support the perception that changes in child quality of life due to multimorbidity are clinically relevant. Given the early onset of multimorbidity, health professionals in the paediatric setting should consider engaging children and families in discussions about mental health soon after the diagnosis of a physical condition is made and discussion surrounding the physical condition completed. Within a holistic family-centred approach, health professionals are encouraged to apply brief screening tools to identify at-risk children and provide referrals to supportive services on a case-by-case basis. This is a critical window of opportunity given that mental disorder is strong predictor of youth suicide ${ }^{69}$ and that risk for suicide is highest soon after an adolescent is diagnosed with a physical condition. ${ }^{34}$ Because of the chronicity and pervasiveness of multimorbidity and its influence on child and parent psychosocial functioning, continual monitoring during routine clinical assessments may also be warranted.

There is one noteworthy limitation: the study was likely underpowered to detect differences between children with and without multimorbidity, and the small sample size may limit the generalisability of findings. However, our sample size was consistent with considerations for 
implementing pilot studies, ${ }^{40}$ and our coverage of eligible families was good.

\section{CONCLUSION}

These preliminary findings indicate that mental disorder in children newly diagnosed with a physical condition is common and negatively affects their quality of life over time. If these results are replicated in a subsequent larger study, health professionals should be aware of the burden of multimorbidity and prepare themselves to discuss mental health with children and their parents. Findings from this pilot study have been used to implement a large-scale study that will examine child multimorbidity in greater depth and provide more definitive clinical implications.

Acknowledgements The authors gratefully acknowledge the children, parents and health professionals and their staff without whose participation this study would not have been possible. We especially thank Jessica Zelman for coordinating the study and Jane Terhaerdt for assisting with ethical approval. Health professional contributors to this study were Janice Falcone, Karen McAssey, Marilyn Rothney, Susan Waserman (McMaster Children's Hospital) and Roberta Berard, Craig Campbell, Margo Devries-Rizzo, Michelle Diebold, Patti Guertjens, Simon Levin and Narayan Prasad (Children's Hospital London Health Sciences).

Contributors MAF led the study. MAF, MHB, KNS, KG, JWG, AG, HLM, ELL and RJVL conceptualised and designed the study and were responsible for acquiring funding. MHB, KNS, KG and AG provided methodological insights. JWG, HLM, ELL and RJVL provided clinical insights. KNS and HLM helped facilitate clinic participation. AB and MAF analysed and interpreted data and drafted the manuscript. All authors critically reviewed and revised and then approved the final manuscript as submitted.

Funding This work was supported by the Canadian Institutes of Health Research (MOP-133645). At the time of the study, MAF was supported by a Research Early Career Award from Hamilton Health Sciences. MAF currently holds the Canada Research Chair in Youth Mental Health, MHB holds the Canada Research Chair in the Social Determinants of Child Health, KG holds the Dan Offord Chair in Child Studies, JWG holds the Scotiabank Chair in Child Health Research, AG is supported by a Canadian Institutes for Health Research New Investigator Award, HLM holds the Chedoke Health Chair in Child Psychiatry and RJVL holds the Canada Research Chair in the Perinatal Programming of Mental Disorders and Albert Einstein/Irving Zucker Chair in Neuroscience.

Competing interests None declared.

Ethics approval The study protocol received ethical approval from the Hamilton Integrated Research Ethics Board (14-130) and Research Ethics Board (105505).

Provenance and peer review Not commissioned; externally peer reviewed.

Data sharing statement Data will not be shared in order to protect the confidentiality of participants. This was a multisite study, and we do not have approval to make the data publicly available.

Open Access This is an Open Access article distributed in accordance with the Creative Commons Attribution Non Commercial (CC BY-NC 4.0) license, which permits others to distribute, remix, adapt, build upon this work non-commercially, and license their derivative works on different terms, provided the original work is properly cited and the use is non-commercial. See: http://creativecommons.org/ licenses/by-nc/4.0/

(c) Article author(s) (or their employer(s) unless otherwise stated in the text of the article) 2018. All rights reserved. No commercial use is permitted unless otherwise expressly granted.

\section{REFERENCES}

1. Van Cleave J, Gortmaker SL, Perrin JM. Dynamics of obesity and chronic health conditions among children and youth. JAMA 2010;303:623-30.
2. van der Lee JH, Mokkink LB, Grootenhuis MA, et al. Definitions and measurement of chronic health conditions in childhood: a systematic review. JAMA 2007;297:2741-51.

3. Merikangas KR, Calkins ME, Burstein M, et al. Comorbidity of physical and mental disorders in the neurodevelopmental genomics cohort study. Pediatrics 2015;135:e927-938.

4. Dobbie M, Mellor D. Chronic illness and its impact: considerations for psychologists. Psychol Health Med 2008;13:583-90.

5. Kessler RC, Avenevoli S, Costello EJ, et al. Prevalence, persistence, and sociodemographic correlates of DSM-IV disorders in the National Comorbidity Survey Replication Adolescent Supplement. Arch Gen Psychiatry 2012;69:372-80.

6. Ferro MA. Major depressive disorder, suicidal behaviour, bipolar disorder, and generalised anxiety disorder among emerging adults with and without chronic health conditions. Epidemiol Psychiatr Sci 2016;25:462-74.

7. Quach J, Barnett T. Impact of chronic illness timing and persistence at school entry on child and parent outcomes: Australian longitudinal study. Acad Pediatr 2015;15:89-95.

8. Canning EH, Hanser SB, Shade KA, et al. Mental disorders in chronically ill children: parent-child discrepancy and physician identification. Pediatrics 1992;90:692-6.

9. Pinquart M, Shen Y. Behavior problems in children and adolescents with chronic physical illness: a meta-analysis. J Pediatr Psychol 2011;36:1003-16.

10. Pinquart $M$, Shen $Y$. Anxiety in children and adolescents with chronic physical illnesses: a meta-analysis. Acta Paediatr 2011;100:1069-76.

11. Pinquart M, Shen Y. Depressive symptoms in children and adolescents with chronic physical illness: an updated meta-analysis. J Pediatr Psychol 2011;36:375-84.

12. Butwicka A, Frisén L, Almqvist $C$, et al. Risks of psychiatric disorders and suicide attempts in children and adolescents with type 1 diabetes: a population-based cohort study. Diabetes Care 2015;38:453-9.

13. Jones LC, Mrug S, Elliott MN, et al. Chronic physical health conditions and emotional problems from early adolescence through midadolescence. Acad Pediatr 2017;17:649-55.

14. Muggah E, Graves E, Bennett C, et al. Ascertainment of chronic diseases using population health data: a comparison of health administrative data and patient self-report. BMC Public Health 2013;13:16.

15. Boyle MH, Duncan L, Georgiades K, et al. Classifying child and adolescent psychiatric disorder by problem checklists and standardized interviews. Int J Methods Psychiatr Res 2017;26:e1544.

16. Rettew DC, Lynch AD, Achenbach TM, et al. Meta-analyses of agreement between diagnoses made from clinical evaluations and standardized diagnostic interviews. Int J Methods Psychiatr Res 2009;18:169-84.

17. Thygesen LC, Ersbøll AK. When the entire population is the sample: strengths and limitations in register-based epidemiology. Eur $J$ Epidemiol 2014;29:551-8.

18. Moreira $\mathrm{H}$, Carona $\mathrm{C}$, Silva $\mathrm{N}$, et al. Psychological and quality of life outcomes in pediatric populations: a parent-child perspective. J Pediatr 2013;163:1471-8.

19. Bai G, Herten MH, Landgraf JM, et al. Childhood chronic conditions and health-related quality of life: Findings from a large populationbased study. PLoS One 2017;12:e0178539.

20. Ferro MA, Boyle MH. Self-concept among children and adolescents with a chronic illness: a meta-analytic review. Health Psychol 2013;32:839-48.

21. Bolognini M, Plancherel B, Bettschart W, et al. Self-esteem and mental health in early adolescence: development and gender differences. J Adolesc 1996;19:233-45.

22. Crump C, Rivera D, London R, et al. Chronic health conditions and school performance among children and youth. Ann Epidemiol 2013;23:179-84.

23. Forrest CB, Bevans KB, Riley AW, et al. School outcomes of children with special health care needs. Pediatrics 2011;128:303-12.

24. Ferro MA, Boyle MH. The impact of chronic physical illness, maternal depressive symptoms, family functioning, and self-esteem on symptoms of anxiety and depression in children. $J$ Abnorm Child Psychol 2015;43:177-87.

25. Miodrag N, Burke M, Tanner-Smith E, et al. Adverse health in parents of children with disabilities and chronic health conditions: a metaanalysis using the parenting stress index's health sub-domain. $J$ Intellect Disabil Res 2015:59:257-71.

26. Newacheck PW, Kim SE. A national profile of health care utilization and expenditures for children with special health care needs. Arch Pediatr Adolesc Med 2005;159:10-17.

27. McCarthy MJ, Behimer G, Anderson JA, et al. Caregiving for youth with co-occurring developmental disabilities and behavioral health 
issues when caregivers face additional health-related stressors: analysis of risk and protective factors from a national sample. Res Dev Disabil 2016;59:399-409.

28. Pinquart M. Parenting stress in caregivers of children with chronic physical condition-a meta-analysis. Stress Health 2017. doi: 10.1002/ smi.2780 [Epub ahead of print 18 Aug 2017].

29. Lee SL, Cheung YF, Wong HS, et al. Chronic health problems and health-related quality of life in Chinese children and adolescents: a population-based study in Hong Kong. BMJ Open 2013;3:e001183.

30. Waters E, Davis E, Nicolas C, et al. The impact of childhood conditions and concurrent morbidities on child health and well-being. Child Care Health Dev 2008;34:418-29.

31. Chen H, Cohen P, Kasen S, et al. Impact of adolescent mental disorders and physical illnesses on quality of life 17 years later. Arch Pediatr Adolesc Med 2006;160:93-9.

32. Wodchis WP, Austin PC, Henry DA. A 3-year study of high-cost users of health care. CMAJ 2016;188:182-8.

33. Anon. Patient- and family-centered care and the pediatrician's role. Pediatrics 2012;129:394-404.

34. Christiansen E, Stenager E. Risk for attempted suicide in children and youths after contact with somatic hospitals: a Danish register based nested case-control study. J Epidemiol Community Health 2012;66:247-53.

35. Speechley KN, Ferro MA, Camfield CS, et al. Quality of life in children with new-onset epilepsy: a 2-year prospective cohort study. Neurology 2012;79:1548-55.

36. Ferro MA, Speechley KN. Examining clinically relevant levels of depressive symptoms in mothers following a diagnosis of epilepsy in their children: a prospective analysis. Soc Psychiatry Psychiatr Epidemiol 2012;47:1419-28.

37. Ferro MA, Van Lieshout RJ, Ohayon J, et al. Emotional and behavioral problems in adolescents and young adults with food allergy. Allergy 2016;71:532-40.

38. Ferro MA, Van Lieshout RJ, Scott JG, et al. Condition-specific associations of symptoms of depression and anxiety in adolescents and young adults with asthma and food allergy. J Asthma 2015:1-26.

39. Miller GF, Coffield E, Leroy Z, et al. Prevalence and costs of five chronic conditions in children. J Sch Nurs 2016;32:357-64.

40. Hertzog MA. Considerations in determining sample size for pilot studies. Res Nurs Health 2008;31:180-91.

41. Sheehan DV, Sheehan KH, Shytle RD, et al. Reliability and validity of the Mini International Neuropsychiatric Interview for children and adolescents (MINI-KID). J Clin Psychiatry 2010;71:313-26.

42. Polanczyk GV, Salum GA, Sugaya LS, et al. Annual research review: a meta-analysis of the worldwide prevalence of mental disorders in children and adolescents. $J$ Child Psychol Psychiatry 2015;56:345-65.

43. Ravens-Sieberer $U$, Auquier P, Erhart M, et al. The KIDSCREEN-27 quality of life measure for children and adolescents: psychometric results from a cross-cultural survey in 13 European countries. Qual Life Res 2007;16:1347-56.

44. Robitail S, Ravens-Sieberer U, Simeoni MC, et al. Testing the structural and cross-cultural validity of the KIDSCREEN-27 quality of life questionnaire. Qual Life Res 2007:16:1335-45.

45. Qadeer RA, Ferro MA. Child-parent agreement on health-related quality of life in children with newly diagnosed chronic health conditions: a longitudinal study. Int J Adolesc Youth 2017:1-10.

46. Berry JO, Jones WH. The parental stress scale: initial psychometric evidence. J Soc Pers Relat 1995;12:463-72.

47. Spielberger CD. State-trait anxiety inventory for adults. Menlo Park: Mind Garden Inc, 1983.

48. Okun A, Stein RE, Bauman LJ, et al. Content validity of the psychiatric symptom index, CES-depression scale, and state-trait anxiety inventory from the perspective of DSM-IV. Psychol Rep 1996;79:1059-69.
49. Radloff LS. The CES-D scale: a self-report depression scale for research in the general population. Appl Psychol Meas 1977;1:385-401.

50. Ferro MA, Speechley KN. Factor structure and longitudinal invariance of the Center for Epidemiological Studies Depression Scale (CES-D) in adult women: application in a population-based sample of mothers of children with epilepsy. Arch Womens Ment Health 2013;16:159-66.

51. Epstein NB, Baldwin LM, Bishop DS. The mcmaster family assessment device*. J Marital Fam Ther 1983;9:171-80.

52. Byles J, Byrne C, Boyle MH, et al. Ontario child health study: reliability and validity of the general functioning subscale of the mcmaster family assessment device. Fam Process 1988;27:97-104.

53. Crichton N. Visual Analogue Scale (VAS). J Clin Nurs 2001;10:706.

54. McCormack HM, Horne DJ, Sheather S. Clinical applications of visual analogue scales: a critical review. Psychol Med 1988;18:1007-19.

55. Paul-Dauphin A, Guillemin F, Virion JM, et al. Bias and precision in visual analogue scales: a randomized controlled trial. Am J Epidemiol 1999;150:1117-27.

56. Couper MP. New developments in survey data collection. Annu Rev Sociol 2017;43:121-45.

57. De Los Reyes A, Kazdin AE. Informant discrepancies in the assessment of childhood psychopathology: a critical review, theoretical framework, and recommendations for further study. Psychol Bull 2005;131:483-509.

58. Jensen PS, Rubio-Stipec M, Canino G, et al. Parent and child contributions to diagnosis of mental disorder: are both informants always necessary? J Am Acad Child Adolesc Psychiatry 1999;38:1569-79.

59. Beck AT, Emery G, Greenberg RL. Anxiety disorders and phobias: a cognitive perspective. New York: Guildford Press, 1985.

60. Hou R, Garner M, Holmes C, et al. Peripheral inflammatory cytokines and immune balance in Generalised Anxiety Disorder: casecontrolled study. Brain Behav Immun 2017;62:212-8.

61. Stein RE, Silver EJ. Operationalizing a conceptually based noncategorical definition: a first look at US children with chronic conditions. Arch Pediatr Adolesc Med 1999;153:68-74.

62. Topal E, Catal F, Soylu N, et al. Psychiatric disorders and symptoms severity in pre-school children with cow's milk allergy. Allergol Immunopathol 2016;44:445-9.

63. Besser MJ, Ganor Y, Levite M. Dopamine by itself activates either D2, D3 or D1/D5 dopaminergic receptors in normal human T-cells and triggers the selective secretion of either IL-10, TNFalpha or both. J Neuroimmunol 2005;169:161-71.

64. Buske-Kirschbaum A, Schmitt J, Plessow F, et al. Psychoendocrine and psychoneuroimmunological mechanisms in the comorbidity of atopic eczema and attention deficit/hyperactivity disorder. Psychoneuroendocrinology 2013;38:12-23.

65. Ferro MA, Boyle MH. Longitudinal invariance of measurement and structure of global self-concept: a population-based study examining trajectories among adolescents with and without chronic illness. J Pediatr Psychol 2013;38:425-37.

66. Ferro MA, Gorter JW, Boyle MH. Trajectories of depressive symptoms during the transition to young adulthood: the role of chronic illness. J Affect Disord 2015;174:594-601.

67. Gonzalez A, Boyle MH, Kyu HH, et al. Childhood and family influences on depression, chronic physical conditions, and their comorbidity: findings from the Ontario child health study. J Psychiatr Res 2012;46:1475-82.

68. Norman GR, Sloan JA, Wyrwich KW. Interpretation of changes in health-related quality of life: the remarkable universality of half a standard deviation. Med Care 2003;41:582-92.

69. Nock MK, Green JG, Hwang I, et al. Prevalence, correlates, and treatment of lifetime suicidal behavior among adolescents: results from the National comorbidity survey replication adolescent supplement. JAMA Psychiatry 2013;70:300-10. 